\title{
¿Qué estamos discutiendo?
}

\author{
analía Gerbaudo / Universidad Nacional del Litoral - CONICET \\ agerbaudo@fhuc.unl.edu.ar; analia.gerbaudo@conicet.gov.ar
}

S'il y a une vérité, c'est que la vérité du monde social est un enjeu de luttes. PIERRE BOURDIEU, «Une classe objet»

Esta editorial se escribe en diálogo con tres notas a las que pretende, además, enviar. La primera, firmada por Jens Andermann para la prestigiosa revista Journal of Latin American Cultural Studies. En su artículo de enero de 20I6, Andermann pone en primer plano a Argentina. Más precisamente, al gobierno de Mauricio Macri y a sus vertiginosas decisiones en materia de políticas públicas. Esas que, como bien repasa el autor, suponen atrocidades que habilitan desde el encarcelamiento de Milagro Sala (9) hasta la liviandad, no meramente discursiva, en el tratamiento de los derechos humanos (en relación con el último punto, Andermann llama la atención sobre el infausto empleo del término "curro" para designar una cuestión tan delicada: su estado de alerta es tal que decide fundar, junto a académicos de Europa y de Estados Unidos, el «Observatorio Argentino» destinado a monitorear el curso de los acontecimientos en el plano de derechos sociales y humanos en nuestro país).

La segunda nota invita a algo más que un mero ejercicio de memoria visual: en su tapa del 6 de diciembre de 20I6, Página/I2 vuelve a estampar la misma foto de Albert Einstein que había estampado en setiembre de 1994 junto al titular: «A lavar los platos». La portada, encabezada por la frase «Otra vez a lavar los platos» se acompańa con datos precisos: «Por los recortes presupuestarios, los llamados a concursos del CONICET para incorporar a nuevos investigadores se redujeron de 943 el ańo pasado a 385 este año, un 60 por ciento menos, al estilo de las políticas de Domingo Cavallo en los 90".

Es notable el contraste entre los datos que traíamos en la editorial del número anterior y que reponía cifras del Banco Mundial respecto del lugar destacado de Argentina entre los países del mundo que más apostaron al sistema científico durante los últimos doce años y estas otras referencias a las que se agregan las 
reportadas en la proliferación de escritos críticos desatados luego de la publicación por el CONICET de los resultados de los Ingresos a la Carrera de Investigador Científico y Tecnológico en diciembre del año pasado. Entre esos ensayos se cuenta la tercera nota a la que envío escrita por Diego Hurtado para la inteligente propuesta intelectual armada desde la Universidad Nacional de San Martín vía la revista Anfibia.

En «Ciencia para qué» este Doctor en Física especializado en historia de la ciencia no sólo da argumentos convincentes para entender la necesidad de las ciencias sociales y humanas en el tejido social sino que historiza el «sinuoso»e inestable devenir del organismo que está en el ojo de la tormenta: el CONICET. De todos los datos que aporta subrayo uno: durante la presidencia de Carlos Menem, un informe del Banco Mundial titulado Argentina. From Insolvency to Growth «recomendaba la privatización del CONICET». Decía este informe: «El CONICET y la Fundación Miguel Lillo deberían ser privatizados, resultando en 5639 posiciones abolidas del presupuesto público». Con lucidez, Hurtado advierte respecto de las "presiones» de los organismos internacionales para que en nuestros países se adopten "formas institucionales, marcos regulatorios y medidas» ajenas a nuestra realidad, a nuestras necesidades, a nuestras proyecciones. Es allí, justamente en este punto, donde destaca la importancia estratégica de un Estado que apueste a las ciencias sociales y humanas porque son estas las que permiten «enfrentar estas presiones» con contra-argumentos junto a propuestas concretas. Es decir, son estas las que aportan «el conocimiento que necesita un Estado para sus políticas públicas». Esas mismas que, como bien recuerda, habían provocado la desafortunada frase de Domingo Cavallo dirigida a la socióloga y demógrafa Susana Torrado cuando esta había cuestionado, durante una entrevista radial, la tasa de desocupación oficial. Más allá de la violencia de género, la sintomática frase expresa mucho más que el modelo de ajuste económico y social del que se derivaba. Como lo revela en una entrevista la propia Torrado: por aquellos ańos «empezaba a hacerse evidente que la actividad científica podía ser útil para el diagnóstico del país». Es decir, útil para un gobierno que quisiera atender a esos resultados. Y sigue: «en los años que van de 1984 a 1994 rehicimos el campo de las ciencias sociales, se recuperó el entramado académico y comienzan a salir al espacio de los medios de comunicación los resultados de los trabajos». Lo que se ataca, entonces, es el potencial crítico de esa información: «el científico no es más ese estereotipo de distraído (...). Su práctica genera información que tiene aspectos sociales y económicos inherentes, que son todo menos abstractos».

En definitiva, vale la pena detenerse a pensar qué estamos discutiendo mientras discutimos, en nuestro caso, y tal como lo venimos haciendo prácticamente desde el primer número de esta revista, los criterios de gestión del conocimiento científico en general, y los criterios específicos para las ciencias sociales y las humanidades, en particular. Algo que, para muchos de los investigadores que fuimos jóvenes en los noventa, se impone como una necesidad imperiosa. Suelo contar, al respecto, una anécdota: en los noventa me presenté en dos ocasiones 
a las becas doctorales del Conicet. Cada año Jorge Panesi firmaba mis papeles como director. Cada ańo recibía la misma carta, entonces vía correo postal, con el invariable resultado: primera o segunda suplente. La cantidad de becas asignadas cabía en dos hojitas A4. Y las del área de Filología, Literatura y Lingüística no superaban el número de dos o tres en todo el país.

Este «cuento» puede entenderse mejor a la luz de otros que salen a la luz a propósito de una mega-investigación que historiza la institucionalización y la internacionalización de las ciencias humanas y sociales en un arco bastante heterogéneo de países y en un período temporal pretencioso. ${ }^{1}$ Retomo aquí parte de un relato de dos críticas literarias, hoy investigadoras del CONICET, sobre aquellos años. Dice Ana Porrúa en una entrevista que concede para el citado proyecto:

En 1992, durante el gobierno de Menem, cuando Matera estaba a cargo de la Secretaría de Ciencia y Técnica, quedé afuera del CONICET, como muchos otros becarios, con un dictamen extemporáneo sobre un informe de Iniciación que ya estaba aprobado; extemporáneo y escandaloso. En ese dictamen, que se publicó parcialmente en algunos diarios (recuerdo una nota en Tiempo Argentino o en Sur, no lo tengo a mano) se decía, entre otras cosas, que yo no hacía crítica literaria sino ideología, porque trabajaba la poesía de Ernesto Cardenal y Roque Dalton. En el mismo año, obtuve una beca de Perfeccionamiento en la Universidad Nacional de Mar del Plata, con la que llevé adelante parte del doctorado.

Cabe llamar la atención sobre la coincidencia con el relato de Adriana Rodríguez Pérsico sobre este mismo punto:

En la actualidad soy Investigadora Principal de CONicet. Los tiempos han cambiado y para bien. Cuando asumió Menem y puso al frente de CONiCET a Matera, se desató una caza de brujas. Muchos quedamos fuera de CONICET y yo emigré.

Por su parte Gustavo Bombini que hacía su doctorado bajo la dirección de Ana María Barrenechea con una beca del ConICET otorgada en I989, la pierde en I99I por motivos parecidos a los de Porrúa. Bombini transforma aquella violencia en escritura y completa su tesis: en 1999 obtiene el título de Doctor en Letras por la Universidad de Buenos Aires. La versión libro de esa investigación terminará ganando, años después, en 2005, el Premio al «mejor libro de educación» de la Fundación El libro en el rubro «Obras teóricas». Paradójico que ese trabajo luego premiado haya sido el mismo que en su versión incipiente había motivado su expulsión del CONICET por usar «bibliografía ideológica» (en verdad, lo que hacía el joven becario era relevar exhaustivamente el estado de la cuestión integrando a sus referencias la ineludible obra de Adriana Puiggrós). Vale la pena, por su contundencia a la hora de retratar las oscilantes e inestables condiciones de producción científica en Argentina, transidas por una improductiva lógica cortoplacista dominante (nadie que haga ciencia y piense seriamente en formar equipos, redes, etc., puede trabajar con miras inmediatas), reponer su «cuento»: 
Con una lógica propia de nuestro país una comisión de notables oscurantistas, ligados a las gestiones científicas de las dictaduras militares, se constituyó en I99I como instancia evaluadora de mis informes de avance en el CONICET del menemismo y con argumentos propios de un autoritarismo poco compatible con la autoridad académica («utiliza bibliografía parcial e ideológica» rezaba — nunca mejor utilizado este verbo— el dictamen redactado por el Dr. Germán Orduna y firmado por algunos miembros de la Comisión, refiriéndose a la monumental Historia de la Educación en la Argentina coordinada por la Dra. Adriana Puiggrós, profesora titular de Historia Latinoamericana y Argentina de la UBA, quien era presentada en el texto de Orduna como un personaje demonizado) evaluó negativamente mis informes y procedió a quitarme el beneficio de la beca doctoral. (19)

También aporta en el esclarecimiento del tipo de constricciones sufridas el testimonio de Ana María Zubieta. Su relato evoca su decisión de apartarse del CONICET al advertir las arbitrariedades y el sectarismo de aquella época:

Ingresé al Conicet en 1986 a Carrera de Investigador porque a pesar de no haber sido becaria, reconocieron mis antecedentes y la ausencia de inserción institucional por los años de dictadura. Renuncié en 1995 por disidencia con procedimientos e ideología de su comisión evaluadora a la cual habían reingresado personas que habían actuado en ella durante la dictadura.

Traigo estos "cuentos» ${ }^{2}$ porque ayudan a reflexionar sobre qué estamos discutiendo mientras discutimos los criterios de gestión de las ciencias sociales y humanas en el marco más expandido de debate sobre las actuales políticas públicas de Argentina. Un debate que se empequeńece tanto si se lo circunscribe sólo al presente o al pasado reciente como si se lo recorta sobre la letra chica de nuestro pequeñísimo «sub-campo», puesto al margen de las vulneraciones cotidianas de derechos en los más variados órdenes.

Por eso es necesario volver al artículo de Andermann: mirándonos desde la distancia que da la extranjería, Andermann lee lo que está en la base de las políticas públicas de esta nueva fase de la posdictadura. ${ }^{3}$ Lee la ideología. No tenemos derecho a ser ingenuos en este punto. Tampoco a des-historizar. No tenemos derecho nosotros: quienes nos reconocemos como investigadores del campo de las ciencias sociales y humanas que, desde este país, asumimos una responsabilidad política y ética, pero fundamentalmente intelectual y laboral con los investigadores por-venir (esos que ahora, todavía, son nuestros becarios del «sistema»).

Es tal vez especialmente por este marco que se vuelve necesario destacar tres motivos para celebrar. El primero: la incorporación a nuestro Comité Científico de Luz Rodríguez Carranza. Junto con su bienvenida, aprovechamos la oportunidad para reiterar nuestro agradecimiento a todos los colegas que nos donan su tiempo al cooperar como evaluadores de nuestra producción. El segundo motivo: la publicación de dos dossiers que condensan resultados de investigación sobre problemáticas que atraviesan las discusiones que se desarrollan en nuestro Centro de 
Investigaciones Teórico-Literarias (nos sucede con la publicación de cada dossier de cada número: que quienes firman los artículos elijan esta revista para poner en circulación estas producciones representa un estímulo invalorable para nuestro trabajo). El tercero: la traducción de un muy reciente artículo de Gisèle Sapiro que contribuye, junto con la entrevista que nos ha concedido, a reponer una versión actualizada de un concepto central para muchas investigaciones del sub-campo de los estudios literarios así como aspectos poco conocidos de su trayectoria intelectual. Estos tres movimientos nos esperanzan: frente a los problemas de diferentes órdenes que jaquean al sistema científico de Argentina, la internacionalización, hasta hace poco objeto de nuestras investigaciones, parece volverse también meta así como fue un movimiento obligado para muchos en otros cortes históricos. Este volverse meta es inescindible de nuestras fantasías ya que no es sino este destino el que parece asegurar-nos un lugar de continuidad de la conversación intelectual, frente al desamparo y la incertidumbre respecto de lo por-venir.

Notas

${ }^{1}$ Hago referencia aquí al proyecto International Cooperation in the Social Sciences and Humanities: Comparative Socio-Historical Perspectives and Future Possibilities (InTERCo ssh, European Union Seventh Framework Programme FP7/2007-2013/ Grant Agreement $\mathrm{N}^{\circ}$ 319974; enero 20I3-febrero 2017). El trabajo sobre la institucionalización y la internacionalización de los estudios literarios, lingüísticos y semióticos (subcampos que integran el campo de las «letras») en Argentina se realizó bajo mi coordinación. En la recolección de los datos participaron inicialmente María Fernanda Alle, Pamela Bórtoli, Cintia Carrió, Daniela Gauna, Ángeles Ingaramo, Micaela Lorenzotti, Sergio Peralta, Lucila Santomero, Ivana Tosti, Santiago Venturini, Carlos Leonel Cherri, Daniela Fumis, Daniel Gastaldello, Silvana Santucci, Gabriela Sierra, Cristian Ramírez y Verónica Gómez: una actividad a concluir en noviembre de 2017. Parte de estos datos como de primeras síntesis parciales están disponibles on line en la página Web del Centro de Investigaciones Teórico-Literarias. A partir de setiembre de 2015 se integran al equipo Nora Catelli, Annalisa Mirizio, Max Hidalgo, Edgardo Dobry y Víctor Escudero (Universidad de Barcelona): sus aportes, centrados en un Estudio comparado de la circulación de la teoría y paradigmas criticos en España y Argentina: academias, conflictos y actores, se condensarán en un Segundo Informe Técnico a publicarse en 2018 en la página Web del mismo centro. Este informe es el segundo de una serie de cinco: el primero, sobre institucionalización (cf. Gerbaudo 20I4); los tres siguientes, centrados en la internacionalización de cada uno de los subcampos referidos (se empieza por el de los estudios literarios, se sigue con el de los estudios lingüísticos y luego con el de los estudios semióticos) y el final proyecta un análisis comparativo de la dinámica de estos subcampos. Este trabajo se enmarca en un nuevo proyecto grupal (Estudios literarios, lingüisticos y semióticos en Argentina: institucionalización e internacionalización 1945-2010, CAI+D UNL, 2017-202I) que incluye además resultados sobre la investigación en Brasil: en marzo de 2017, a partir de la presentación de avances en la Universidade Federal de Santa Catarina, se incorporó al colectivo Maria Lúcia de Barros Camargo y su equipo.

2 Para comprender cómo se articulan las cuestiones de orden cualitativo alrededor de este concepto con los resultados de orden cuantitativo propiciados por la mega-investigación referida, ver el artículo donde expongo sus bases categoriales (2017).

${ }^{3}$ En mi caracterización del término hice hincapié en el carácter relativo de su inestable «fin» en 2003 junto al estado "por-venir» de nuestras democracias (cf. 20I6). 


\section{Bibliografía}

andermann, Jens (2016). "It's The Ideology, Stupid! In Macri's Argentina: A Reportage». Journal of Latin American Cultural Studies 25(I), 7-17.

вомвINI, gUSTAVo (2004). Los arrabales de la literatura. La historia de la enseñanza literaria en la escuela secundaria argentina (I860-1960). Buenos Aires: Miño y Dávila/ubA.

Gerbaudo, ANALÍA (Dir.) (20I4). La institucionalización de las Letras en la universidad argentina (1945-2010). Notas "en borrador» a partir de un primer relevamiento [en línea]. Santa Fe: Universidad Nacional del Litoral. Consultado el 6 de abril de 2017 en http://www.fhuc.unl. edu.ar/centros/cedintel/interco_vf.pdf

(2016). Politicas de exhumación. Las clases de los criticos en la universidad argentina de la posdictadura (1984-1986). Los Polvorines/Santa Fe: Universidad de General Sarmiento/ Universidad Nacional del Litoral.

(2017). "Derivas conceptuales (un borrador)». IV Coloquio de avances de investigaciones del CEDINTEL [en línea]. Santa Fe: Universidad Nacional del Litoral. Consultado el 6 de abril de 2017 en http://www.fhuc.unl.edu.ar/centros/cedintel/interco_vf.pdf

hurtado, diego (20I6). "Ciencia para qué». Anfibia [en línea]. Consultado el 6 de abril de 2017 en http://www.revistaanfibia.com/ensayo/ciencia-para-que/ PORRÚa, ANA (2OI5). Entrevista por Santiago Venturini. INTERCo SSH-EHESS/CAI+D-UNL. RODRÍGUeZ PÉRSICO, ADRIANa (2016). Entrevista por Pamela Bórtoli e Ivana Tosti. INTERCo SSH-EHESS/CAI+D-UNL.

torrado, susana (2009, 27 de setiembre). «Lavar los platos» [en línea]. Página/I2. Consultado el 6 de abril de 2017 en https:/www.paginaı2.com.ar/diario/suplementos/ futuro/13-2222-2009-09-27.html ZUBIETA, ANA MARÍA (2OI6). Entrevista por Cristian Ramírez. INTERCo SSH-EHESS/CAI+D-UNL. 